P22 (continued)

Objective: To understand the impacts of the COVID-19 pandemic on youth handwashing and washing produce before eating behaviors.

Study Design, Setting, Participants: Data were collected from youth $(\mathrm{N}=1,098)$ in grades $3-5$ who participated in SNAP-Education (SNAP-Ed) and the Expanded Food and Nutrition Education Program (EFNEP) in Florida. Utilizing common self-report survey measures, baseline measures from 3 years prior to the pandemic and 3 months during the pandemic were compared for handwashing before eating and washing produce before eating. Due to incomplete data collection during the first 8 months of the pandemic, only behaviors from October 20-January 31 intervals (October 2018- January 2021) were compared to control for seasonal differences.

Measurable Outcomes/Analysis: Behavioral measures are based on a frequency scale from 1 to 4 (4 being the most frequent). An independent samples $t$ test was used to compare baseline FSP scores from intervals before and during the pandemic.

Results: No significant difference was found in the frequency of handwashing before and during COVID-19 (3.16 vs 3.18, respectively; $P=0.76$ ). A significant increase was found in the frequency of washing produce before eating before and during COVID-19 (3.14 vs 3.31, respectively; $P=0.008$ ).

Conclusion: This study did not find any significant changes in handwashing frequency during COVID-19, however, a significant increase in washing produce prior to eating was found. Handwashing prior to eating is a current CDC guideline for COVID-19 mitigation while washing produce prior to eating is not (CDC 2021). Changes in lifestyle and/or eating arrangements during the pandemic might explain these findings.

Funding: Supplemental Nutrition Assistance Program Education.

\section{P23 Dietary Changes Among Japanese Adults Since the Spread of COVID-19}

Misa Shimpo,PhD, RD, shimpo.misa@u-nagano.ac.jp, The University of Nagano, 8-49-7, Miwa, Nagano, Nagano, Japan, 380-8525; Rie Akamatsu, RD, DrPH, Ochanomizu University; Yui Kojima, $P h D, R D$, University of Niigata Prefecture

Background: Preventive measures adopted for COVID19 have brought about significant changes in lifestyle and have affected dietary habits worldwide.

Objective: To examine the factors relating to dietary changes among Japanese adults since the spread of COVID-19.

Study Design, Settings, Participants: A cross-sectional questionnaire survey was conducted via the internet in November 2020. All 6,000 participants, comprising 3,044 male and 2,956 female adults, aged 20-64 years, who registered with a research company received the questionnaire on email.

Measurable Outcome/Analysis: The questionnaire included items on demographic characteristics; socioeconomic factors; past medical history; COVID-19 infection situation of their family and neighbors; fear of COVID-19; and lifestyle habits and healthy changes in dietary habits since the spread of COVID-19. The participants were given multiple choices in answers: changed to healthier; changed to unhealthier; and no-change. Multinomial logistic regression analysis was conducted to examine factors associated with dietary changes.

Results: Of the participants, 1,215 (20.3\%) answered that their dietary habits became healthier, 491 (8.2\%) answered as unhealthy, while a majority of them $(4,294$; $71.6 \%)$, said their dietary habits remained unchanged. The results of the multinomial logistic regression analysis with unchanged as the reference indicated that healthier and unhealthier dietary change were negatively associated with age (OR [95\% CI]: 0.89[0.84-0.95], 0.89 [0.81-0.97]) and positively associated with the past medical history of dyslipidemia (1.44 [1.11-1.86], 1.74 [1.23-2.46]) and fear of COVID-19 (1.05 [1.04-1.07], 1.02 [1.01-1.04]). Unhealthy change was positively associated with living alone, COVID-19 infection of colleagues, stress, and BMI; while annual household income, COVID-19 infection of oneself and friends, health literacy, frequency of exercise, and smoking were positively associated with healthier dietary changes.

Conclusion: This study suggested that the factors determining healthy and unhealthy dietary change since the spread of COVID-19 were age, COVID-19 infection, and fear of COVID-19.

Funding: Health, Labour and Welfare Policy Research Grants, Special Research (Grant Number JP 20CA2040).

\section{P24 Effect of a Nudge Intervention for Increasing Vegetable Intake Among University Students in a University Cafeteria}

Makiko Nakade,PhD, RD, nakade@shse.u-hyogo.ac.jp, University of Hyogo; Research Institute for Food and Nutritional Sciences, 1-1-12 Shinzaike-honcho, Himejicity, Hyogo, Japan, 6700092; Mako Matsushita, University of Hyogo; Ryota Fukui, BS, University of Hyogo; Kokoro Nakamura, University of Hyogo; Miho Fujishiro, University of Hyogo

Background: An intake of $\geq 350$ g of vegetables per day is recommended in Japan. However, the average intake of vegetables among adults $\geq 20$ years is about $280 \mathrm{~g}$ in 2019 , and the intake of young adults in their 20 s, including university students, is low (about $223 \mathrm{~g}$ ). Recently, the use of nudge strategies for behavioral change has been gaining attention. However, relatively few nudge trials for increasing vegetable intake have been conducted in Japan. 